\title{
Screening the Missing Electron: Nanochemistry in Action
}

\author{
H. Shiozawa, ${ }^{1}$ T. Pichler, ${ }^{2}$ C. Kramberger, ${ }^{2}$ M. Rümmeli, ${ }^{3}$ D. Batchelor, ${ }^{4}$ Z. Liu, ${ }^{5}$ \\ K. Suenaga, ${ }^{5}$ H. Kataura, ${ }^{6}$ and S. R.P. Silva ${ }^{1}$ \\ ${ }^{1}$ Advanced Technology Institute, University of Surrey, Guildford, GU2 7XH, United Kingdom \\ ${ }^{2}$ Fakultät für Physik, Universität Wien, Strudlhofgasse 4, 1090 Wien, Austria \\ ${ }^{3}$ IFW Dresden, D-01171 Dresden, Germany \\ ${ }^{4}$ Universität Würzburg, BESSY II, D-12489 Berlin, Germany \\ ${ }^{5}$ Research Center for Advanced Carbon Materials, AIST, Tsukuba 305-8565, Japan \\ ${ }^{6}$ Nanotechnology Research Institute, AIST, Tsukuba 305-8562, Japan
}

(Received 14 September 2008; published 27 January 2009)

\begin{abstract}
The excitement of nano-test-tube chemistry in a single-walled carbon nanotube is exemplified in our study on electron doping in carbon nanotubes. Electron doping through the 1D van Hove singularity of single-walled carbon nanotubes is realized via a chemical reaction of an encapsulated organocerium compound, $\mathrm{CeCp}_{3}$. The decomposition of $\mathrm{CeCp}_{3}$ inside the carbon nanotubes increases the doping level and greatly enhances the density of conduction electrons. The transition of the cerium encapsulating semiconducting tubes to metallic results in enhanced screening of the photoexcited core hole potential. This fact illustrates the importance of many body effects in understanding core-level excitation process in carbon nanotubes.
\end{abstract}

DOI: 10.1103/PhysRevLett.102.046804

PACS numbers: 73.22.-f, 78.30.-j, 78.70.Dm, 79.60.-i

A single-walled carbon nanotube (SWCNT) can be conceptualized as a rolled up graphene sheet [1]. The quantum confinement of electrons along the tube circumference yields a rich variety in conductivities with the chirality determining its metallic or semiconducting nature [2]. The electronic structure is intimately related to its atomic configuration [3-6]. In order for carbon nanotubes to fulfill their promise as electronic materials, that encompass their properties such as ballistic conduction [7] and high current density [8,9], their synthesis, purification, and functionalization need to be addressed. Remarkable progress in their purification, such as the separation of metallic and semiconducting tubes, has taken place $[10,11]$. Besides such chirality separations, doping of SWCNTs with atoms or molecules is a promising means to tune their bulk electronic properties. Doping through the van Hove singularity (VHS) can efficiently modulate the conductivity of SWCNTs [12,13]. Electron doping of SWCNT bundles by alkali metal intercalation results in a transition from a Tomonaga-Luttinger liquid to a Fermi liquid [14,15]. The excellent structural and chemical integrity of carbon nanotubes provides an ideal 1D environment with which to explore chemistry at the nanoscale [16-24]. Such nanochemistry allows tweaking the doping level of encapsulating metallic SWCNTs [25,26]. These facts highlight the versatility of SWCNTs as a fundamental component for electronic devices and as a route for the quest for improved understanding in chemistry and solid state physics.

In this Letter, we report on the doping of SWCNTs by cerium compounds. Tris(cyclopentadienyl)cerium $\left(\mathrm{CeCp}_{3}\right)$, which consists of three cyclopentadienyl rings
(Cp), bound to a central cerium atom, is used as a precursor for cerium encapsulation within SWCNTs. We demonstrate that the encapsulated chemical reaction enables electron doping through the VHS of SWCNT.

Clear evidence for filling is provided by Raman spectroscopy and transmission electron microscopy (HRTEM), both of which show the transition of the encapsulated $\mathrm{CeCp}_{3}$ into innershell tubes upon annealing. On the conversion, electron doping of the encapsulating semiconducting tube is observed as vanishing VHS peaks in the C $1 s$ absorption spectra and as the appearance of the carbon Fermi edge in the valence-band photoemission spectra. Moreover, two additional features appear near the $\mathrm{C} 1 s$ absorption onset at high doping, which we attribute to the VHS peaks of the original semiconducting tubes with enhanced core hole screening due to their transition to metallic tubes.

The SWCNT material was synthesized by laser ablation and purified by an $\mathrm{H}_{2} \mathrm{O}_{2}$ treatment [27]. The purified tube material and $\mathrm{CeCp}_{3}$ powder were sealed in an evacuated Pyrex tube and annealed up to $200{ }^{\circ} \mathrm{C}$ for $24 \mathrm{~h}$. After this process the tube material was thoroughly rinsed by sonicating in acetone multiple times. Finally, a bucky film was peeled off the filter. Raman spectroscopy was carried out using a Bruker FT Raman spectrometer with a wavelength of $1064 \mathrm{~nm}$. X-ray absorption experiments were conducted at the synchrotron beam line UE 52 PGM, Bessy II. Either drain current or partial electron yield was collected with an overall resolving power better than 10000 . Resonance photoemission spectroscopy was performed by using a hemispherical SCIENTA SES 200 photoelectron energy 
analyzer. The experimental resolution and the Fermi energy were determined from the Fermi edge of an in situ deposited Au film or clean molybdenum substrate. The base pressure in the setup was maintained below $5 \times$ $10^{-10}$ mbar.

The left-hand panel of Fig. 1 shows the Raman spectra of a $\mathrm{CeCp}_{3}$ filled SWCNT sample before and after annealing at $1000^{\circ} \mathrm{C}$ in vacuum. Both spectra contain the expected radial breathing modes of pristine tubes at frequencies ranging from 130 to $210 \mathrm{~cm}^{-1}$. After annealing, sharp lines appear in the range of $300-370 \mathrm{~cm}^{-1}$, corresponding to the formation of inner tubes and, hence, evidence $\mathrm{CeCp}_{3}$ encapsulation within the SWCNT. HRTEM observations provide us further insight into the chemical identity of the filling. The right-hand top panel of Fig. 1 confirms a typical micrograph of the $\mathrm{CeCp}_{3} @ \mathrm{SWCNT}$ in which arrays of contrast corresponding to encapsulated $\mathrm{CeCp}_{3}$ molecules are easily discerned within the tube walls. The middle and bottom panels show HRTEM micrographs of the sample transformed into double-walled carbon nanotubes (DWCNT) containing cerium $(\mathrm{Ce} @ \mathrm{DWCNT})$. The undulating lines observed in between the outer tube wall indicate an inner tube, while the dark dots are from cerium ions.

Resonance photoemission spectroscopy can extract element specific electronic information of compounds. With a photon energy in resonance with the Ce core absorption edge, the partial electronic states of cerium are predominantly observed in the valence-band photoemission spectrum. Conversely, when off resonance, the nanotube carbon valence states dominate in our case. In Fig. 2(a), we compare the Ce $3 d \rightarrow 4 f$ on-resonance photoemission spectra of the pristine and annealed $\mathrm{CeCp}_{3}$ filled
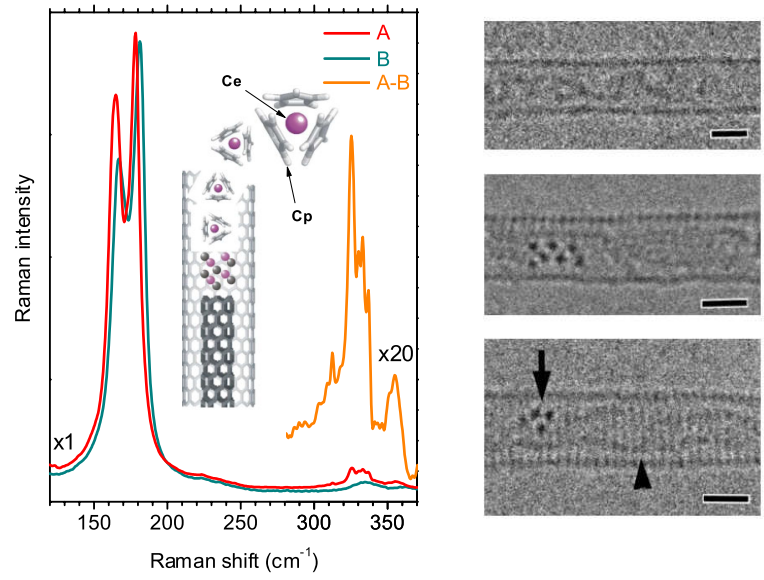

FIG. 1 (color online). Left: Raman spectra for Ce@DWCNT $(A), \mathrm{CeCp}_{3} @ \operatorname{SWCNT}(B)$, and their difference $(A-B)$ at the radial breathing mode region. The inset shows a schematic model for the conversion from the encapsulated $\mathrm{CeCp}_{3}$ molecules to the innershell tube. Right: TEM micrographs of $\mathrm{CeCp}_{3} @ \mathrm{SWCNT}$ (top) and Ce@DWCNT (middle and bottom). Scale bar is $1 \mathrm{~nm}$.
SWCNT. Both spectra are, as a whole, very similar to each other and exhibit a peak corresponding to the $\mathrm{Ce}$ $4 f^{1} \rightarrow 4 f^{0}$ transition at a binding energy of $2 \mathrm{eV}$. In contrast, the off-resonance spectra exhibit striking differences as shown in Fig. 2(b). After annealing, the photoemission intensities near the Fermi level are significantly increased. This is better observed from the $\mathrm{Ce} 4 d \rightarrow 4 f$ resonance at a higher resolution. Expanding the $\mathrm{Ce} 4 d \rightarrow 4 f$ offresonance spectrum close to the Fermi energy region in the main panel of Fig. 2(c), one can easily see the Fermi edge for the annealed sample (Ce@DWCNT). The fact that there is no corresponding Ce $4 f$ peak observed in the offresonance spectra validates the off-resonance condition both at the Ce $3 d$ and $4 d$ edges. This confirms that the changes are associated with the Fermi edge of the carbon valence band. Electron doping of SWCNT bundles up to the $S_{1}^{\star}$ level induces a transition from the TomonagaLuttinger liquid state to the Fermi liquid state due to an increase in tube-tube interactions within bundles, as was shown for potassium intercalated SWCNT [15]. Our case can therefore be interpreted as electron doping filling states above the $S_{1}^{\star}$ level and provides direct evidence for the enhanced conductivity of the SWCNT bundles by cerium doping. This doping takes place equally in the filled SWCNTs, but not in the unfilled SWCNTs, enabling selective doping which is crucial for understanding the following analysis of the $\mathrm{C} 1 s$ excitations.

Figure 3(a) shows the $\mathrm{x}$-ray absorption spectra for pristine and annealed $\mathrm{CeCp}_{3}$ filled SWCNT at the $\mathrm{C} 1 s \rightarrow \pi^{\star}$ excitation region. The pristine $\mathrm{CeCp}_{3} @ \mathrm{SWCNT}$ shows discernible fine structures corresponding to the unoccupied $S_{1}^{\star}, S_{2}^{\star}, M_{1}^{\star}$, and $S_{3}^{\star}$ VHS peaks, very similar to empty SWCNT [25,28]. Upon annealing the $S_{1}^{\star}$ and $S_{2}^{\star}$ are significantly suppressed due to electron doping of the semi-
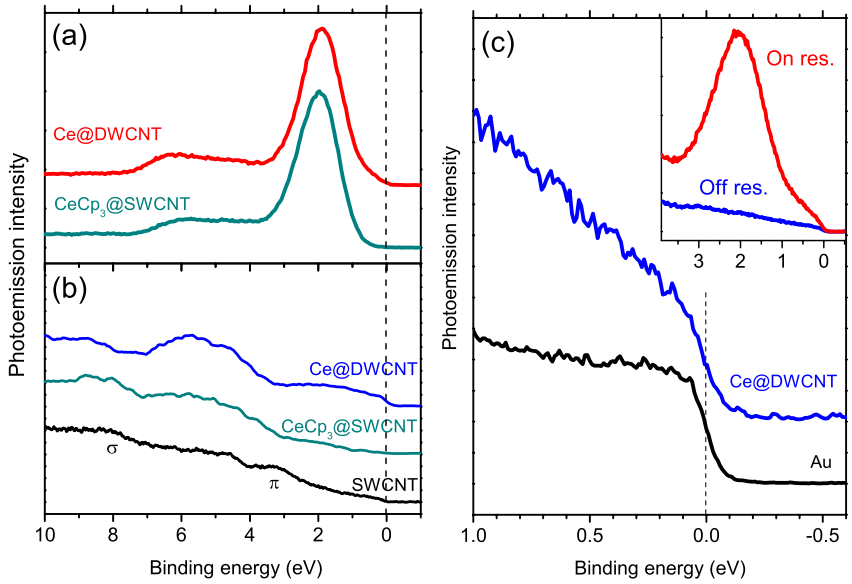

FIG. 2 (color online). (a) Ce $3 d$ on-resonance photoemission spectra obtained at a photon energy of $884 \mathrm{eV}$. (b) Ce $3 d$ offresonance photoemission spectra obtained at $875 \mathrm{eV}$. The reference spectrum of SWCNT was taken at $400 \mathrm{eV}$. (c) Ce $4 d$ offresonance photoemission spectrum near the Fermi level in comparison to that for a clean $\mathrm{Au}$. Inset: $\mathrm{Ce} 4 d$ on- and offresonance photoemission spectra. 
conducting tubes [19]. Furthermore, examining the low energy tail of the $\mathrm{C} 1 s$ absorption edge, two structures can be observed near the onset of the $\pi^{\star}$ peak for the Ce@DWCNT [indicated by the vertical bars, $S_{1}^{M \star}$ and $S_{2}^{M \star}$. Importantly, the appearance of these two structures coincides with the disappearance of the $S_{1}^{\star}$ and $S_{2}^{\star}$ peaks. From this fact, it is likely that these two features have the same origin, i.e., a sudden energy leap of the $S_{1}^{\star}$ and $S_{2}^{\star}$ peaks to the $S_{1}^{M \star}$ and $S_{2}^{M \star}$. The corresponding C $1 s$ photoemission spectra are shown in Fig. 3(b). The main peak of $\mathrm{CeCp}_{3} @ \mathrm{SWCNT}$ is located at a binding energy of $284.6 \mathrm{eV}$, the same as for the pristine SWCNT. In contrast, the $\mathrm{C} 1 \mathrm{~s}$ peaks for the annealed samples show significant energy shifts to lower binding energies, that are about $0.1 \mathrm{eV}$ after annealing at $800^{\circ} \mathrm{C}$ for $2 \mathrm{~h}$ (intermediate) and $0.3 \mathrm{eV}$ for the Ce@DWCNT. The inset shows clearly that this monotonic energy shift is triggered by annealing. The C $1 s$ absorption spectrum of Ce@DWCNT is composed of the $\pi$ and $\sigma$ structures of $s p^{2}$ carbon, essentially very similar to the pristine SWCNT. From this information it is possible to exclude chemical shifts. Such energy shifts can occur when the photoabsorption final state energy is lowered. The final state energy of the core-level photoemission process is dominated by the screening of the core hole potential. In general, the more conduction electrons available, the stronger the expected screening. In our case, the enhanced conductivity by doping can yield stronger screening of the core hole potential, which explains the lower energy of the C $1 s$ photoemission peak for the Ce@DWCNT sample.

In turn, upon $\mathrm{C} 1 s \rightarrow \pi^{\star}$ near edge absorption of a SWCNT, an incoming photon excites one electron from the $\mathrm{C} 1 s$ core level to the unoccupied $\pi^{\star}$ state, as illustrated in the left-hand panel of Fig. 3(c). The final state of this process includes one hole $\left(h^{+}\right)$at the $\mathrm{C} 1 s$ and one electron $\left(e^{-}\right)$at the previously unoccupied state. In the case of semiconducting tubes, excitons are the quasiparticles which lower the energy of the excited state. When the semiconducting tubes become metallic, the excitons are no longer stable for a reasonable lifetime. From this picture, the final state of the doped semiconducting tubes, now metallic, should increase in energy. This is the opposite of what is observed. Some other effect lowering the final energy state with a reasonable energy scale is required.

Core hole screening can once again be applied to explain the above observation. In the core absorption process, both the excited electron and other available conduction electrons are responsible for screening the created core hole in the final state. The absorption threshold energy depends on the availability of conduction electrons [29]. For semiconducting tubes, the photoexcited electrons are responsible for screening the core hole (self-screening). If such a tube couples with the adjacent metallic tubes, the core hole can effectively be screened by the conduction electrons (metal screening). It has been reported that the interactions between different chirality tubes, especially between the semiconducting and metallic tubes, are rather weak so that the Tomonaga-Luttinger liquid state can be observed in metallic tubes in bundles at low temperatures [14]. This means self-screening should dominate metal screening in semiconducting tubes in a bundle. When these semiconducting tubes become metallic by doping, self-screening is no longer dominant due to the strong scattering of the excited electrons in the conduction band. Instead, all conduction electrons are now responsible for the core hole screening. In this case the incident is a many body effect over the whole conduction band and the screening effect becomes much stronger so as to lower the total energy of the photoexcited electron system.

In the right-hand panel of Fig. 3(c), the results of a peak fit analysis of the $\mathrm{C} 1 s$ absorption spectra are presented.
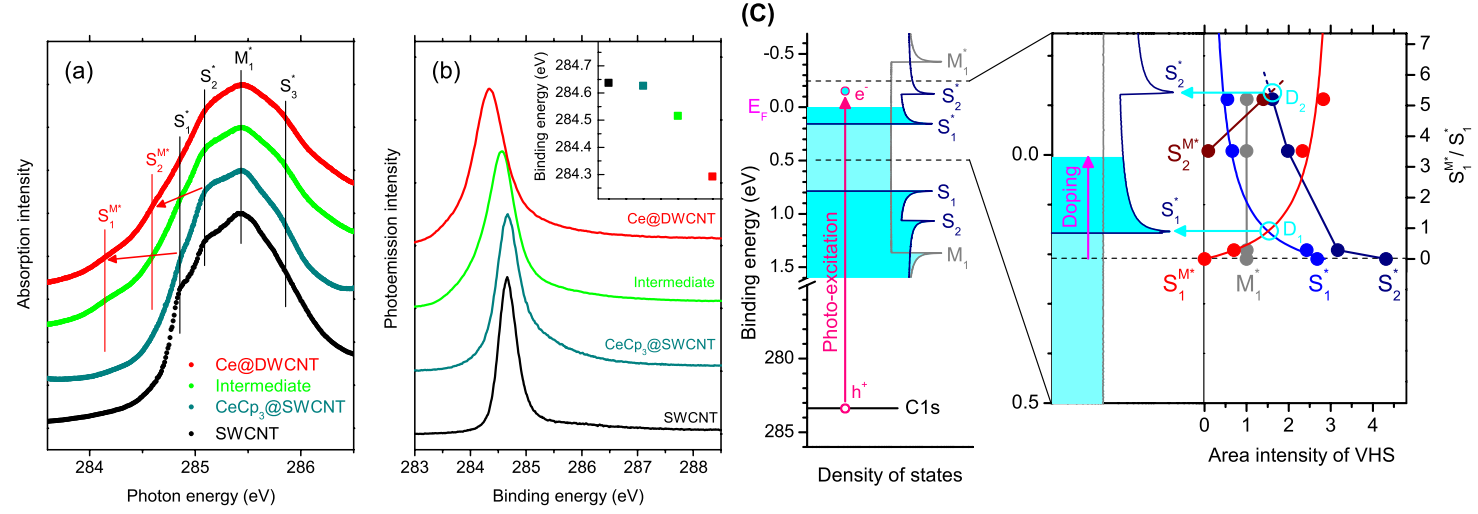

FIG. 3 (color online). (a) C $1 s \rightarrow \pi^{\star}$ absorption. (b) C $1 s$ photoemission. Inset shows C $1 s$ main peak positions. (c) Left: Schematic diagram for C $1 s \rightarrow \pi^{\star}$ photoabsorption process. Right: Normalized area intensity of van Hove singularity absorption peaks, $S_{1}^{\star}$ (blue), $S_{2}^{\star}$ (navy), $M_{1}^{\star}$ (gray), $S_{1}^{M \star}$ (red), and $S_{2}^{M \star}$ (brown), as a function of the intensity ratio of $S_{1}^{M \star} / S_{1}^{\star}$. The vertical arrow indicates chemical-reaction-induced doping of the conduction states of tubes. The blue and red curves are the results of fitting for the $S_{1}^{\star}$ and $S_{1}^{M \star}$ using the equations $f_{1}(\alpha)=C(1+\alpha)^{-1}$ and $f_{2}(\alpha)=C \alpha(1+\alpha)^{-1}$, respectively, where $C=$ const and $\alpha=S_{1}^{M \star} / S_{1}^{\star}$. The open circles, $D_{1}$ and $D_{2}$, indicate the crossing points between $S_{n}^{\star}$ and $S_{n}^{M \star}(n=1,2)$ and correspond to the doping levels at the $S_{1}^{\star}$ and $S_{2}^{\star}$ peaks in the density of states, respectively. 
The area intensities of the VHS peaks, $S_{1}^{\star}, S_{2}^{\star}, M_{1}^{\star}, S_{1}^{M \star}$, and $S_{2}^{M \star}$, were normalized against the $M_{1}^{\star}$ intensity and plotted as a function of the intensity ratio $S_{1}^{M \star} / S_{1}^{\star}$. In this case, the vertical axis, $S_{1}^{M \star} / S_{1}^{\star}$, represents the doping level of the tubes increased by $\mathrm{CeCp}_{3}$ filling and subsequent annealing. It shows that the $S_{1}^{M \star}$ peak can be resolved even for pristine $\mathrm{CeCp}_{3} @ \mathrm{SWCNT}$. The presence of electron doping from $\mathrm{CeCp}_{3}$ to the tubes can be expected based on a previous study on ferrocene filled SWCNT. Upon annealing further, suppression of the $S_{1}^{\star}$ and $S_{2}^{\star}$ peak intensities concomitant with the rising intensities of the $S_{1}^{M \star}$ and $S_{2}^{M \star}$ peaks are observed. Assuming that the sum of the $S_{1}^{\star}$ and $S_{1}^{M \star}$ is constant and $S_{1}^{\star}+S_{1}^{M \star}=C$, we obtain a simple equation for the $S_{1}^{\star}$ and $S_{1}^{M \star}$ intensities, $f_{1}(\alpha)=$ $C(1+\alpha)^{-1}$ and $f_{2}(\alpha)=C \alpha(1+\alpha)^{-1}$, respectively, where $\alpha=S_{1}^{M \star} / S_{1}^{\star}$. The corresponding data fit well to these functions [see the $S_{1}^{\star}$ (blue) and $S_{1}^{M \star}$ (red) curves in the right-hand panel of Fig. 3(c)]. This strongly supports our assignment for the $S_{n}^{M \star}(n=1,2)$ peaks. At the crossing point of these two curves, the $S_{1}^{\star}$ and $S_{1}^{M \star}$ have the same intensity. At this doping level half the original $S_{1}^{\star}$ peak is filled. This idea should also hold for the $S_{2}^{\star}$ peak and is illustrated in the right-hand panel of Fig. 3(c), where the crossing points, $D_{1}$ and $D_{2}$, are correlated to the $S_{1}^{\star}$ and $S_{2}^{\star}$ peaks in the density of states.

This analysis is consistent with the sudden energy leap in VHS peaks from semiconducting tubes as a consequence of the transition to a doping induced metallic phase. Since the semiconducting gap and chemical potential depend on the chirality of the tubes, doping of each semiconducting tube is not a simultaneous event. This is the reason why both the $S_{1}^{M \star}$ and $S_{2}^{M \star}$ peaks are observed at moderate doping levels.

$\mathrm{CeCp}_{3}$ encapsulating SWCNT is used as a template for nanochemistry to achieve doping through the VHS of encapsulating SWCNT. From an extended element specific analysis using resonance core-level spectroscopy, we provide evidence for massively enhanced conductivity of doped SWCNT. The enhanced density of conduction electrons heightens core hole screening of the initial semiconducting tubes. This fact illustrates the importance of many body effects in carbon nanotubes. Nanotest-tube chemistry provides for the use of 1D quantized electronic levels of carbon nanotubes to manipulate physical properties and monitor the chemical process in a confined space. Hence, this work opens a pathway for challenging fundamental issues in correlated 1D electronic systems.

We thank EPSRC, U.K., for financial support through a Portfolio Partnership grant. H.S. acknowledges the Alexander von Humboldt Foundation. C. K. acknowledges the IMPRS. M.H.R. acknowledges the DFG (PI440/4). H.K. acknowledges the Ministry of Education, Culture, Sports, Science and Technology of Japan for funding via a Grant-in-Aid for Scientific Research A (No. 18201017).
[1] S. Iijima, Nature (London) 354, 56 (1991).

[2] M.S. Dresselhaus, G. Dresselhaus, and P.C. Eklund, Science of Fullerenes and Carbon Nanotubes (Academic, New York, 1997).

[3] A. M. Rao et al., Science 275, 187 (1997).

[4] M. S. Dresselhaus, Nature (London) 391, 19 (1998).

[5] J.W. G. Wildöer, L.C. Venema, A. G. Rinzler, R.E. Smalley, and C. Dekker, Nature (London) 391, 59 (1998).

[6] C. E. Giusca, Y. Tison, V. Stolojan, E. Borowiak-Palen, and S. R. P. Silva, Nano Lett. 7, 1232 (2007).

[7] A. Javey, J. Guo, Q. Wang, M. Lundstrom, and H. Dai, Nature (London) 424, 654 (2003).

[8] R.H. Baughman, A.A. Zakhidov, and W. A. de Heer, Science 297, 787 (2002).

[9] Z. Wu et al., Science 305, 1273 (2004).

[10] R. Krupke, F. Hennrich, H.v. Löhneysen, and M. M. Kappes, Science 301, 344 (2003).

[11] M. S. Arnold, A. A. Green, J.F. Hulvat, S. I. Stupp, and M. C. Hersam, Nature Nanotech. 1, 60 (2006).

[12] R. S. Lee, H. J. Kim, J.E. Fischer, A. Thess, and R.E. Smalley, Nature (London) 388, 255 (1997).

[13] A. M. Rao, P.C. Eklund, S. Bandow, A. Thess, and R.E. Smalley, Nature (London) 388, 257 (1997).

[14] H. Ishii et al., Nature (London) 426, 540 (2003).

[15] H. Rauf, T. Pichler, M. Knupfer, J. Fink, and H. Kataura, Phys. Rev. Lett. 93, 096805 (2004).

[16] J. Lee et al., Nature (London) 415, 1005 (2002).

[17] D. J. Hornbaker, S.-J. Kahng, S. Misra, B. W. Smith, A. T. Johnson, E. J. Mele, D. E. Luzzi, and A. Yazdani, Science 295, 828 (2002).

[18] J. Sloan, A.I. Kirkland, J.L. Hutchison, and M.L.H. Green, Chem. Commun. (Cambridge) 13 (2002) 1319.

[19] T. Takenobu, T. Takano, M. Shiraishi, Y. Murakami, M. Ata, H. Kataura, Y. Achiba, and Y. Iwasa, Nature Mater. 2, 683 (2003).

[20] L. J. Li, A. N. Khlobystov, J. G. Wiltshire, G. A. D. Briggs, and R. J. Nicholas, Nature Mater. 4, 481 (2005).

[21] Y. Maniwa, K. Matsuda, H. Kyakuno, S. Ogasawara, T. Hibi, H. Kadowaki, S. Suzuki, Y. Achiba, and H. Kataura, Nature Mater. 6, 135 (2007).

[22] Z. Liu, K. Yanagi, K. Suenaga, H. Kataura, and S. Iijima, Nature Nanotech. 2, 422 (2007).

[23] T. Pichler, Nature Mater. 6, 332 (2007).

[24] H. Shiozawa, T. Pichler, A. Grüneis, R. Pfeiffer, H. Kuzmany, Z. Liu, K. Suenaga, and H. Kataura, Adv. Mater. 20, 1443 (2008).

[25] H. Shiozawa et al., Phys. Rev. B 77, 153402 (2008).

[26] H. Shiozawa, T. Pichler, R. Pfeiffer, and H. Kuzmany, in Nanomaterials: Inorganic and Bioinorganic Perspectives, edited by C. M. Lukehart and R. A. Scott (John Wiley \& Sons, Ltd., Chichester, U.K, 2008), pp. 129-148.

[27] H. Kataura, Y. Maniwa, M. Abe, A. Fujiwara, T. Kodama, K. Kikuchi, H. Imahori, Y. Misaki, S. Suzuki, and Y. Achiba, Appl. Phys. A 74, 349 (2002).

[28] C. Kramberger, H. Rauf, H. Shiozawa, M. Knupfer, B. Buchner, T. Pichler, D. Batchelor, and H. Kataura, Phys. Rev. B 75, 235437 (2007).

[29] J. Stöhr, NEXAFS Spectroscopy (Springer-Verlag, Berlin, 1992). 